Revue pluridisciplinaire d'études médiévales

\title{
La Bible anglo-normande: traduction et adaptation à la croisée de facteurs socioculturels
}

\section{Tatiana Romashkina}

\section{(2) OpenEdition}

\section{Journals}

Édition électronique

URL : http://journals.openedition.org/questes/4846

DOI : 10.4000/questes.4846

ISSN : 2109-9472

Éditeur

Les Amis de Questes

Édition imprimée

Date de publication : 1 juillet 2018

Pagination : 43-62

ISSN : 2102-7188

\section{Référence électronique}

Tatiana Romashkina, « La Bible anglo-normande : traduction et adaptation à la croisée de facteurs socioculturels », Questes [En ligne], 38 | 2018, mis en ligne le 18 juin 2018, consulté le 21 avril 2019 URL : http://journals.openedition.org/questes/4846 ; DOI : 10.4000/questes.4846 


\section{La Bible anglo-normande : traduction et adaptation à la croisée de facteurs socioculturels}

\section{Tatiana ROMASHKINA}

Université de Strasbourg

La Bible anglo-normande occupe une place singulière parmi les Bibles médiévales en raison du contexte spatio-temporel particulier de sa composition. Elle fait son apparition en Angleterre, pays qui, à la suite de la conquête normande de 1066, est devenu l'un des premiers et principaux centres de production manuscrite en français ${ }^{1}$. Datée du $\mathrm{XIV}^{\mathrm{e}}$ siècle, elle apparaît pendant «l'âge d'or de la diffusion des Bibles [...] françaises ${ }^{2} \gg$. Les conditions de sa composition contribuent à sa dimension spécifique qui joue à la fois sur la tradition et l'innovation. La Bible anglo-normande propose une traduction quasiment complète ${ }^{3} \mathrm{de}$ la Vulgate en prose: cette manière de présenter le texte biblique ne

\footnotetext{
${ }^{1}$ Geneviève Hasenohr, «Les origines monastiques », dans Mise en page et mise en texte du livre manuscrit, dir. Henri-Jean Martin et Jean Vezin, Paris, Éditions du Cercle de la Librairie/Promodis, 1990, p. 231-234, voir en particulier p. 231. Pour plus d'informations sur les rapports entre l'anglo-normand et le français médiéval, voir David Trotter, «Not as Eccentric as it Looks: Anglo-French and French French », Forum for Modern Language Studies, vol. 39, 2003, p. 427-438.

${ }^{2}$ Pierre-Maurice Bogaert, «La Bible française au Moyen Âge », dans Les Bibles en français. Histoire illustrée du Moyen Âge à nos jours, dir. Pierre-Maurice Bogaert, Turnhout, Brepols, 1991, p. 13-46, cit. p. 30.

${ }^{3}$ La Bible anglo-normande nous est parvenue à travers trois témoins. Le manuscrit BnF fr. 1 peut être considéré comme presque complet : il s'interrompt au milieu du chapitre XIII de l'Épître aux Hébreux. Le manuscrit British Library Royal 1.C.III traduit les premiers livres bibliques, de la Genèse à Tobie. Enfin, le BnF fr. 9562 présente le Livre des Actes des Apôtres.
} 
commence à se développer qu'à partir de $1250^{4}$. En même temps, la Bible anglo-normande s'inscrit dans une tradition qui a vu le jour dans l'espace insulaire avant l'expédition de Guillaume le Conquérant. Il s'agit d'une intention essentiellement didactique de translatio : la traduction du texte sacré est adaptée et accompagnée de gloses ${ }^{5}$. Les manuscrits BnF fr. 1 et British Library Royal 1.C.III ${ }^{6}$ de la Bible anglo-normande en sont l'illustration ${ }^{7}$. Le premier, composé entre le milieu et le troisième quart du XIV ${ }^{\mathrm{e}}$ siècle $^{8}$, fait partie des collections du Département des Manuscrits de la Bibliothèque Nationale de France; le second, qui remonte à la première moitié ou au milieu du $\operatorname{XIV}^{\mathrm{e}}$ siècle $^{9}$, est conservé dans les collections occidentales de la British Library. Ces manuscrits montrent un vrai contraste non seulement dans leur mise en page, mais aussi dans leur

\footnotetext{
${ }^{4}$ Pierre-Maurice Bogaert, «La Bible française au Moyen Âge », art. cit., p. 27-30. Les transpositions de la Bible datées du XII ${ }^{\mathrm{e}}$ et $\mathrm{du}$ XIII $^{\mathrm{e}}$ siècle ne proposent que des visions partielles de certains livres (ibid., p. 16-17). En effet, «la chronologie [...] confirme grosso modo l'antériorité des vers sur la prose, des traductions partielles sur les traductions complètes, de l'adaptation et de l'Histoire Sainte sur la traduction littérale du texte biblique » (ibid., p. 14).

${ }^{5}$ Pierre Nobel, «Gloses anglaises et latines dans une traduction biblique anglonormande (ms. Londres British Library Royal I.C.III) », dans «Si a parlé par moult ruiste vertu ». Mélanges de littérature médiévale offerts à Jean Subrenat, dir. Jean Dufournet, Paris, Champion, coll. «Colloques, congrès et conférences sur le Moyen Âge », 2000, p. 419-435.

${ }^{6}$ Abrégés par la suite en «manuscrit de Paris » et «manuscrit de Londres ».

${ }^{7}$ Le troisième témoin de la Bible anglo-normande, le BnF fr. 9562, composé dans la deuxième moitié du XIV ${ }^{\mathrm{e}}$ siècle (Pierre-Maurice Bogaert, «La Bible française au Moyen Âge », art. cit., p. 33), ne sera pas présenté dans cette étude car son texte pose des problèmes spécifiques qui ne permettent pas d'analyser cette copie dans une perspective comparative : il s'agit de questions de fond (divergence des contenus), mais également de forme (omissions de mots, de phrases).

${ }^{8}$ François Avril et Patricia Danz Stirnemann, Manuscrits enluminés d'origine insulaire (VII $-X X^{e}$ siècle), Paris, Bibliothèque nationale, coll. «Manuscrits enluminés de la Bibliothèque nationale », 1987, p. 157.

${ }^{9}$ D'après Ruth J. Dean, Anglo-Norman Literature. A guide to texts and manuscripts, cité par Pierre Nobel, «La Bible anglo-normande et la Bible d'Acre: question de source », dans L'Histoire littéraire, ses méthodes et ses résultats. Mélanges offerts à Madeleine Bertaud, dir. Luc Fraisse, Genève, Droz, coll. "Histoire des idées et critique littéraire », 2001, p. 429-448, voir p. 430.
} 
langue et dans leur traduction, directement liées à des facteurs socioculturels.

Cette étude vise donc à présenter et analyser les divergences entre le BnFfr. 1 et le British Library Royal 1.C.III qui doivent illustrer l'influence du public visé, mais également du scribe sur l'élaboration du manuscrit et de son texte. Les exemples proviennent des cinq premiers chapitres de notre édition synoptique en cours ${ }^{10}$ du Premier Livre de Samuel $^{11}$. Il s'agira d'étayer certaines caractéristiques dégagées dans des études antérieures sur le Livre de la Genèse ${ }^{12}$, comme les questions de mise en page qui ont permis de différencier les destinataires des deux copies.

Dans cette optique, nous mettrons en parallèle l'aspect extérieur des deux manuscrits. Puis, nous procéderons à une étude qualitative des traits sémantiques qui fera ressortir la figure des destinataires de ces copies et leur influence sur le travail de traduction accompli par des scribes ; nous nous attarderons sur les éléments, relevant des domaines de la graphie, de la phonétique et de la syntaxe, qui permettront de découvrir les conditions de réalisation de ces $\operatorname{copies}^{13}$.

\footnotetext{
${ }^{10}$ Le travail d'édition synoptique du Premier Livre de Samuel de la Bible anglonormande fait partie intégrante de notre thèse de doctorat, provisoirement intitulée Traduire la Bible au XIV siècle: l'expression de la beauté. Ces recherches s'effectuent sous la direction de Thierry Revol, Professeur en langue et littérature françaises du Moyen Âge à l'université de Strasbourg.

${ }^{11}$ Dans les manuscrits, cette partie est intitulée Regum Primus (ms. de Londres, «le Premier des Rois »)/Liber Regum (ms. de Paris, «le Livre des Rois ») d'après la division ancienne de la Septante.

12 Thierry Revol, Bible anglo-normande. Genèse : édition, université Marc BlochStrasbourg II (en cours de publication).

${ }^{13}$ Les publications de Pierre Nobel, «Gloses anglaises et latines... », art. cit., et « Les translateurs bibliques et leur public : l'exemple de la Bible d'Acre et de la Bible anglo-normande », Revue de linguistique romane, vol.66, 2002, p.451-472, ont permis de mesurer le contraste entre les manuscrits de Londres et de Paris, qui réside dans la façon de traduire; il s'agit notamment des questions du maniement des langues présentes dans les copies par des scribes et, surtout, des destinataires visés.
} 


\section{Mise en page et mise en texte : étude des éléments divergents}

À la fin du XIX ${ }^{\mathrm{e}}$ siècle, dans le cadre des études sur la Bible anglonormande, le manuscrit de Paris a été qualifié, pour la première fois, de livre de famille ${ }^{14}$. Preuve en est la présence dans ce texte de nombreux blasons de familles nobles, y compris celle de John de Welles ${ }^{15}$, pour laquelle cette copie a été confectionnée ${ }^{16}$.

D'autres éléments du décor ont incité les médiévistes à accorder à ce manuscrit une place exceptionnelle parmi les copies de la Bible anglonormande ${ }^{17}$. La première page du Premier Livre de Samuel dans le manuscrit de Paris ${ }^{18}$ est ornée d'une épaisse guirlande de ramages qui sépare les deux colonnes du texte: elle se prolonge sur la marge inférieure et sur la marge supérieure, jusqu'à déborder sur le bord extérieur droit et à entourer les premiers versets du livre. Un bout-deligne décoratif marque la fin de la partie précédente. Le début du Premier Livre de Samuel est signalé par une enluminure: cette tendance est visible dans tout le manuscrit ${ }^{19}$. Les miniatures des livres bibliques sont

Ces découvertes ont été étayées par l'édition du premier livre de la Bible anglonormande (Thierry Revol, Bible anglo-normande..., éd. cit.).

${ }^{14}$ Samuel Berger, La Bible française au Moyen Âge. Étude sur les plus anciennes versions de la Bible (écrites en prose de langue d'oül) [1884], Genève, Slatkine, 1967, p. 303.

${ }^{15}$ Fils d'Adam de Welles (1304-1345), John de Welles (1334-1361) est le quatrième baron de Welles. Voir Nicholas Harris Nicolas, A synopsis of the peerage of England. Exhibiting, under alphabetical arrangement, the date of creation, descent, and present state of every title or peerage which has existed in this country since the Conquest, London, J. Nichols and Son, 1825, vol. 2, p. 680.

${ }^{16}$ Samuel Berger, La Bible française au Moyen Âge..., éd. cit., p. 324.

17 « Ce manuscrit porte le numéro 1 dans les fonds français de la Bibliothèque nationale; c'est assez dire que son volume et sa singularité, sinon sa beauté, lui donnent une place exceptionnelle », ibid., p. 230.

${ }^{18}$ Figure 1 - Première page du Premier Livre de Samuel, Paris, BnF fr. 1 (fol. 78v). Voir infra, p. 60.

19 « Travaillant apparemment sans modèle, l'artiste de fr. 1 a forgé une iconographie nominaliste fort intéressante à partir du début du texte de chaque livre », François Avril et Patricia Danz Stirnemann, Manuscrits enluminés d'origine insulaire (VII ${ }^{e}$ $X X^{e}$ siècle), op. cit., p. 159. 
généralement aussi larges que les colonnes du texte et se trouvent à l'intérieur des lettres initiales, qu'on appelle, de ce fait, «lettres historiées ». La grande initiale du Premier Livre de Samuel ${ }^{20}$ représente une scène d'offrande : en haut au centre, se trouvent deux prêtres portant la barbe et vêtus de l'aube à bordure d'orfroi ; divers dons leur sont tendus par trois hommes à gauche. Ces deux personnages centraux sont deux fils d'Éli recevant le sacrifice offert par Elchana et sa famille ${ }^{21}$. Le soin apporté à la page liminaire du Premier Livre de Samuel dans le BnF fr. 1 ne fait que confirmer tout ce qui a été révélé dans des études antérieures sur ses destinataires ${ }^{22}$ : le manuscrit a donc bien été réalisé pour des milieux aristocratiques. Sa présentation visuelle établit un net contraste avec celle du British Library Royal 1.C.III.

Les motifs décoratifs dans le manuscrit de Londres $^{23}$ se trouvent majoritairement concentrés sur les initiales. Ce sont des lettres filigranées ornées de cadeaux, comme par exemple l'initiale $U^{24}$, rehaussée de motifs ondulés et de traits fins qui se prolongent vers le haut et le bas de la page. L'intérieur de la lettre initiale du Premier Livre de Samuel avec un fond à petits carreaux noirs est occupé par un dessin symétrique rappelant les motifs d'une fleur superposée sur l'image d'une croix rhombique. Dépourvue d'autres éléments visuellement frappants, la mise en page du début du Premier Livre de Samuel dans le manuscrit de Londres est ainsi

\footnotetext{
${ }^{20}$ Figure 2 - Grande initiale du Premier Livre de Samuel, Paris, BnF fr. 1 (fol. $78 v^{\circ}$ b). Voir infra, p. 61.

${ }^{21}$ François Avril et Patricia Danz Stirnemann, Manuscrits enluminés d'origine insulaire (VII $-X X^{e}$ siècle), op. cit., p. 157. Nous gardons la transcription des noms propres proposée par les auteurs de cet ouvrage; de ce fait, elle sera différente de celle dans la traduction de la Vulgate (voir infra, « Héli »).

${ }^{22}$ Voir Samuel Berger, La Bible française au Moyen Âge..., op. cit. ; Pierre Nobel «Les translateurs bibliques et leur public...», art. cit.; Thierry Revol, Bible anglonormande..., éd. cit.

${ }^{23}$ Figure 3 - Première page du Premier Livre de Samuel, Londres, British Library Royal 1.C.III (fol. 186v). Voir infra, p. 61.

${ }^{24}$ Figure 4 - Grande initiale du Premier Livre de Samuel, Londres, British Library Royal 1.C.III (fol. $186 v^{\circ}$ a). Voir infra, p. 62.
} 
plus sobre que celle du manuscrit de Paris. D'après Pierre Nobel, «il ne s'agit assurément pas là d'un exemplaire de qualité destiné à un grand personnage ou une famille importante. Son aspect eût été différent ${ }^{25} \gg$. La simplicité de la copie laisse donc supposer que le manuscrit constitue une Bible de clerc ${ }^{26}$.

Si l'on s'intéresse à la matérialité de l'écriture elle-même, il faut souligner deux types totalement différents: alors que le manuscrit de Paris est copié en belle écriture pâle, le manuscrit de Londres présente une cursive anglaise rapide plutôt «relâchée, sans grande tenue ${ }^{27}$ ». Cependant, le contenu textuel de ce dernier témoigne du travail très rigoureux et minutieux du scribe. La répartition des corrections dans les deux manuscrits permet d'illustrer le propos :

\begin{tabular}{|c|c|c|}
\hline $\begin{array}{c}\text { Nombre de } \\
\text { corrections... }\end{array}$ & $\begin{array}{c}\text { Manuscrit de } \\
\text { Londres }\end{array}$ & Manuscrit de Paris \\
\hline du copiste & $21(81 \%)$ & $9(39 \%)$ \\
\hline $\begin{array}{c}\text { dans le travail } \\
\text { d'édition }\end{array}$ & $5(19 \%)$ & $14(61 \%)$ \\
\hline Total & $26(100 \%)$ & $23(100 \%)$ \\
\hline
\end{tabular}

Tableau - Répartition des corrections dans les manuscrits ${ }^{28}$ (Premier Livre de Samuel, I-V)

Le nombre total de corrections dans les cinq premiers chapitres du Premier Livre de Samuel ( $c f$. Tableau) montre que le copiste du manuscrit de Londres commet un peu plus de fautes que le scribe de celui de Paris. Cependant, ses propres interventions sur le texte $(81 \%)$ prouvent que le scribe accorde une grande attention au travail de copie. Il procède par

\footnotetext{
${ }^{25}$ Pierre Nobel, «Les translateurs bibliques et leur public... », art. cit., p. 453.

26 «L'absence de destinataire clairement identifié plaide en faveur de cet usage anonyme dans le cadre d'un monastère ou d'une communauté religieuse », Thierry Revol, Bible anglo-normande..., éd. cit., p. 16.

${ }^{27}$ Pierre Nobel, « Les translateurs bibliques et leur public... », art. cit., p. 452.

${ }^{28}$ Les résultats ont été obtenus dans les cinq premiers chapitres du Premier Livre de Samuel, édités dans le cadre de nos travaux de thèse. Durant le comptage, nous avons distingué les corrections scribales de nos propres corrections éditoriales pour pouvoir analyser la méthode de travail de deux scribes avec le texte.
} 
exponctuation dans les cas où il faut soustraire certains termes ou graphèmes :

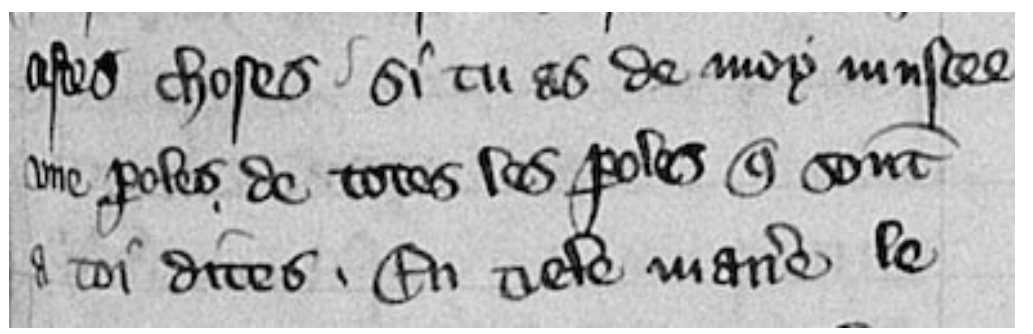

Correction par exponctuation (voir $2^{\mathrm{e}}$ ligne : parole), Londres, British Library Royal 1.C.III (fol. 189r $\left.{ }^{\circ} a\right)$.

Dans les cas où il faut ajouter des lettres ou des mots manquants, il réalise une insertion interlinéaire :

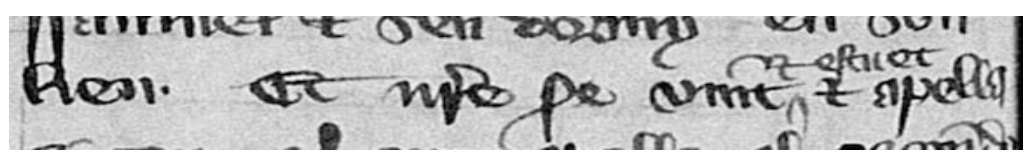

Correction par insertion interlinéaire (et estuet), Londres, British Library Royal 1.C.III (fol. $188 v^{\circ}$ b).

L'insertion s'effectue généralement avec un signe qui rappelle la lettre grecque lambda en majuscule. Cette approche soigneuse apparaît encore plus évidente lors de la comparaison avec le travail du scribe du manuscrit de Paris, beaucoup plus inattentif et distrait : $61 \%$ de ses fautes restées sans corrections ont été révélées lors du travail d'édition (cf. Tableau).

Il existe encore un indice saillant qui permet d'établir un parallèle entre cette attention extrême au texte, que montre le copiste du manuscrit de Londres $^{29}$, et son intention essentiellement didactique : il s'agit de l'insertion de gloses. En effet, le manuscrit de Londres porte sur la première page de garde une mention latine datée $\mathrm{du} X \mathrm{~V}^{\mathrm{e}}$ siècle qui précise sa localisation à l'abbaye de Reading: «Hic est liber monachorum claustralium Radingie $^{30} \gg$. Bien que le lieu précis de sa composition reste

\footnotetext{
${ }^{29}$ Une telle approche est très probablement due à la signification particulière du corpus (texte sacré) pour le scribe.

${ }^{30}$ The British Library, Explore Archives and Manuscripts (Catalogue en ligne), URL: <http://searcharchives.bl.uk> (moteur de recherche Explore Archives and
} 
inconnu, les traces laissées par le copiste font donc bien penser à une Bible de travail pour un clerc.

Dans l'édition du Livre de la Genèse ${ }^{31}$, les gloses du manuscrit de Londres sont présentées en deux langues, le latin et l'anglais, ce qui permet de faire l'hypothèse de l'origine insulaire du copiste. Dans les cinq premiers chapitres édités du Premier Livre de Samuel, on rencontre les trois gloses suivantes ${ }^{32}$ (toutes en $\operatorname{latin}^{33}$ ) : «(II, 14) Et l'envoia en le caudroun (lebetem), ou en caudre (caldarium), ou en pot, ou en caudroun (cacabum); et tote la rien que le havet levoit, tolli le chapellain a soi $[\ldots] »$. Ce sont les termes synonymiques concrets de la source (lebetem, caldariam $^{34}$, [ollam], cacabum ${ }^{35}$ ) qui posent problème au

Manuscripts), <http://goo.gl/z84k71> (raccourci d'URL avec Google URL Shortener, page consultée le 28 mars 2017) ; Pierre Nobel, «La Bible anglo-normande et la Bible d'Acre...», art. cit., p. 431. Nous traduisons: «C'est un livre des moines claustraux de Reading ».

${ }^{31}$ La Genèse du manuscrit de Londres comporte au total 47 gloses anglaises et 27 gloses latines (Thierry Revol, Bible anglo-normande..., éd. cit., p. 72-73).

${ }^{32}$ Voir infra. Les gloses des cinq premiers chapitres du Premier Livre de Samuel se trouvent toutes dans le même verset 14 du chapitre II.

${ }^{33}$ Dans les deux exemples (voir infra), l'italique indique les mots en question et/ou leurs gloses. Le scribe du manuscrit de Londres utilise le soulignement pour les gloses, maintenu dans notre travail d'édition et également conservé dans l'exemple. Cependant, l'italique utilisée pour la transcription des unités abrégées dans l'édition a été enlevée, pour plus de clarté.

${ }^{34}$ Cette forme féminine se trouve dans deux versions actuelles de la Vulgate : Biblia Sacra iuxta latinam Vulgatam Versionem. Liber Samuhelis, Roma, Typis Polyglottis Vaticanis, 1944, p. 79, et Biblia Sacra iuxta Vulgatam Versionem [1969], éd. Roger Gryson et Robert Weber, Stuttgart, Deutsche Bibelgesellschaft, 1994, p. 368. Le scribe du manuscrit de Londres utilise pourtant la forme neutre. En effet, les dictionnaires latins admettent les deux genres pour le sens «chaudron » (Félix Gaffiot, Dictionnaire latin-français, Paris, Hachette, 1934, p. 243), « chaudière » (Charles du Fresne du Cange et al., Glossarium medice et infima latinitatis, Niort, L. Favre, 1883-1887, URL: <http://ducange.enc.sorbonne.fr>, <http://ducange.enc.sorbonne.fr/CALDARIA\#CALDARIA-7> [page consultée le 28 mars 2017]).

${ }^{35}$ Biblia Sacra iuxta latinam Vulgatam Versionem. Liber Samuhelis, éd. cit., p. 79. Ce texte qui comporte un grand nombre de variantes, de même que Biblia Sacra iuxta Vulgatam Versionem (éd. cit.), sont deux éditions de base dans le travail d'édition du Premier Livre de Samuel. Nous traduisons : «chaudière, chaudron, marmite, pot» (d'après La Sainte Bible selon la Vulgate [1902], trad. Jean-Baptiste Glaire, Argentrédu-Plessis, D.F.T., 2002, p. 515). 
copiste. Il reprend donc les termes de la Vulgate, afin de corriger ou peutêtre de nuancer et d'assurer sa traduction française. Il les insère dans le fil du texte, en les distinguant par un trait de soulignement :

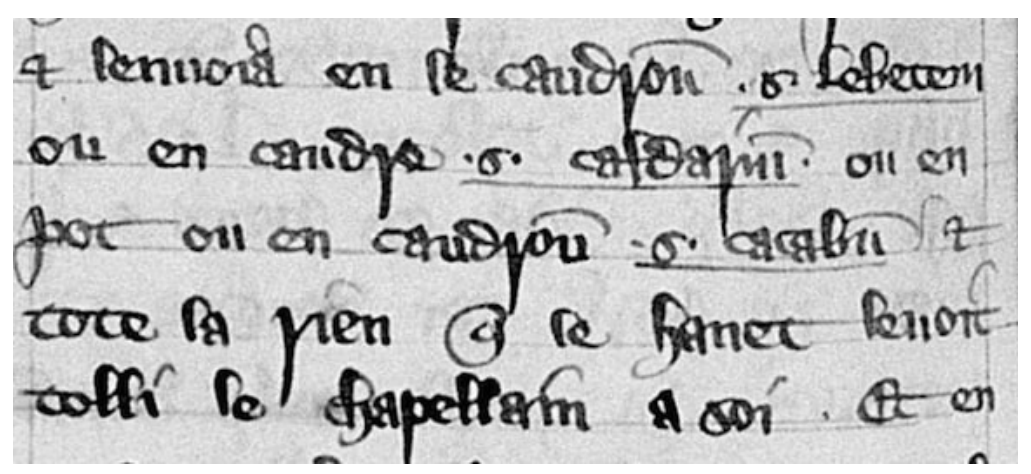

Gloses latines, Londres, British Library Royal 1.C.III (fol. $187 \mathbf{v}^{\circ} \mathbf{b}$ ).

Le passage du copiste au latin semble assez naturel : les moines et les clercs lisent plus facilement que les laïques la Bible en cette langue qu'ils ont étudiée pendant leur formation. Et si le scribe du manuscrit de Paris procède par omission des termes de la Vulgate qui manquent en français - « (II, 14) Et l'envoia en caudroun, ou en chaudere, ou en poot; et tote la rien que le crok levoit, tolli le chapellain a soi $[\ldots] \gg-$, le copiste du manuscrit de Londres traduit les quatre termes latins avec deux fois le même mot (caudroun). De ce fait, il n'omet rien malgré le manque de variété dans les termes, mais en utilisant les gloses, il transmet le plus fidèlement possible le texte biblique ${ }^{36}$.

\section{Contexte et conditions de la production manuscrite à travers les indices linguistiques}

Quoique la mise en page et la mise en texte jouent un rôle important dans la description des manuscrits médiévaux, c'est surtout le texte des copies qui est valorisé pour lui-même dans les études du

\footnotetext{
36 «Le ms. $L$ présente une translation mot à mot de la Bible, au point que le texte français résiste parfois à la compréhension sans le secours de la source latine. Si cette servilité est voulue, elle peut être l'expression d'un souci d'exactitude. C'est ce que manifesterait aussi la présence des gloses latines ou anglaises », Pierre Nobel, «Gloses anglaises et latines... », art. cit., p. 434.
} 
français médiéval. Les données linguistiques peuvent contribuer aux connaissances en médiévistique de façon totalement transdisciplinaire.

Ainsi le choix sémantique des copistes semble-t-il être parfois dépendant du public visé. Les versets 8 et 11 du chapitre $v$ du Premier Livre de Samuel illustrent le contraste remarquable entre deux copies dans la traduction du mot latin satrapes ${ }^{37}$ :

${ }^{8}$ et mittentes congregaverunt omnes satrapas Philisthinorum ad se et dixerunt ${ }^{38}[\ldots]$

$\mathrm{ML}^{39}:(\mathrm{V}, 8)$ Et cil envoiaunt, assemblerent a soi touz les sages des Philistiens, et distrent [...]

$\mathrm{MP}^{40}$ : $(\mathrm{V}, 8)$ Et cil envoiauntz, assemblerent a soi tuz les princes des Philystiens, et distrent $[\ldots]$

${ }^{11}$ miserunt itaque et congregaverunt omnes satrapas Philisthinorum qui dixerunt ${ }^{41}[\ldots]$

ML : (v, 11) Por ceo envoierent il et assemblerent touz les sages mesters de Philistiens $[\ldots]$

MP : $(\mathrm{V}, 11)$ Et cil envoierent en tiele manere et assemblerent tuz les princes des Phylistiens [...]

Bien que le terme satrape soit déjà utilisé en français à l'époque de la composition des manuscrits de la Bible anglo-normande ${ }^{42}$, les copistes

\footnotetext{
${ }^{37}$ Nous avons mis en italique la traduction du mot satrapes dans les exemples qui suivent.

${ }^{38}$ I Samuel, v, 8, Biblia Sacra iuxta Vulgatam Versionem, éd. cit., p. 373, «et ils envoyèrent chercher, et rassemblèrent tous les satrapes des Philistins auprès d'eux, et dirent [...] » (La Sainte Bible selon la Vulgate, éd. cit., p. 521).

${ }^{39}$ Manuscrit de Londres.

${ }^{40}$ Manuscrit de Paris.

${ }^{41}$ I Samuel, v, 11, Biblia Sacra iuxta Vulgatam Versionem, éd. cit., p. 373, «c'est pourquoi ils envoyèrent chercher et rassemblèrent tous les satrapes des Philistins, qui dirent [...]»(La Sainte Bible selon la Vulgate, éd. cit., p. 521).

${ }^{42}$ Ce mot apparaît dans le Trésor de Brunet Latin avec un sens de «gouverneur d'une province chez les Perses » (Centre National de ressources textuelles et lexicales, Étymologie, URL : 〈http://www.cnrtl.fr >, 〈http://www.cnrtl.fr/etymologie/satrape> [page consultée le 28 mars 2017]). Le Dictionnaire du Moyen Français, URL : <http://www.atilf.fr/dmf/> (page consultée le 9 mai 2017), en donne d'autres exemples au XIV siècle : voir Guillaume de Machaut, «Le Confort d'ami », dans
} 
lui préfèrent des termes différents ${ }^{43}$ : sage (sage mester) dans le manuscrit de Londres et prince dans le manuscrit de Paris ${ }^{44}$. Même s'ils peuvent entretenir des liens d'équivalence sémantique ${ }^{45}$, un choix lexical divergent figure dans les deux copies. Le terme prince, attesté à partir de la première moitié du XII ${ }^{\mathrm{e}}$ siècle avec les sens de «celui qui possède une souveraineté » ou encore de «celui qui est le principal personnage d'un groupe » dans le Psautier d'Oxford ${ }^{46}$, semble désigner dans le manuscrit «celui qui détient l'autorité, chef, supérieur ${ }^{47}$ ». Le substantif sage, présenté dans le manuscrit de Londres au verset 8 , apparaît dans les textes

Guillaume de Machaut, Euvres, éd. Ernest Hoepffner, Paris, Champion, 1921, vol. 3, p. 1-42, cit. p. 36, et Philippe de Mézières, Le Songe du vieil pelerin, éd. George William Coopland, Cambridge, Cambridge University Press, 1969, vol. 2, p. 473 (nous remercions pour cette suggestion Monsieur Claude Buridant, Professeur émérite à l'université de Strasbourg). Voir aussi la note suivante sur la présence de ce terme dans le manuscrit de Londres.

${ }^{43}$ Cependant, la suite du texte (I Samuel, VI, 5 ; VI, 12 ; VI, 16 ; VII, 7) montre que le scribe de Londres recourt à ce terme, qui fait toujours partie de la même expression « satrapes des Philistins ».

${ }^{44}$ La suite du texte prouve la même tendance pour le copiste de Paris. Pourtant, il remplace exceptionnellement le terme prince par consaillour (au pluriel) dans le verset 5 du chapitre VI.

${ }^{45}$ Le Dictionnaire du Moyen Français, URL: <http://www.atilf.fr/dmf/> (page consultée le 9 mai 2017), illustre ainsi le sens de «sage» («celui qui sait conseiller, guider ») par un exemple issu du «Confort d'ami » de Guillaume de Machaut, éd. cit., p. 18, où les deux termes se côtoient et se trouvent dans une relation apparemment synonymique : «Li rois riches dons li donna /Et maistre et signeur l'ordonna /Et fist de toutes les provinces/Sus les sages et sus les princes/De son païs de Babiloine./Adont Daniel sans essoine,/Pour bien et par le gré dou roy,/Pour gouverner de bon arroy /Les provinces, y mist Sydrac ».

${ }^{46}$ Psautier d'Oxford, II, 2 et LXVII, 30, éd. Francisque Michel, cité dans Centre National de ressources textuelles et lexicales, Étymologie, URL : <http://www.cnrtl.fr/etymologie/prince> (page consultée le 9 mai 2017).

47 L'exemple proposé par le Dictionnaire du Moyen Français, URL : <http://www.atilf.fr/dmf/> (page consultée le 9 mai 2017), est daté de 1366 : «Sacrefie a noz diex, /Et de noz prestres en touz lieux /Le maistre et le prince seras » («Miracle de saint Ignace», dans Miracles de Nostre Dame par personnages, éd. Gaston Paris et Ulysse Robert, Paris, Didot, 1879, vol. 4, p. 71-118, cit. p. 80). L'exemple sert d'illustration de la synonymie possible entre prince et maistre (rappelons que ce dernier terme est proposé dans la copie de Londres). 
dès 1100 , où il signifie « celui qui a sa raison, son bon sens ${ }^{48}$ » ou « celui qui a une connaissance juste des choses, qui est le représentant de la sagesse » (1181-1190) ${ }^{49}$. Le sens du mot dans le manuscrit semble correspondre à « celui qui sait conseiller, guider ${ }^{50} »$. L'adjectif $\operatorname{sage}^{51}$, épithète de mester au verset 11, est employé dans son acception de « savant, instruit ${ }^{52}$ », « éclairé ${ }^{53}$ », alors que le substantif qui fait partie du groupe nominal ${ }^{54}$ correspond à « personne qui exerce une autoritét ${ }^{55} »$. De ce fait, le manuscrit de Londres apparemment destiné au milieu spirituel et clérical (voir supra) semble transmettre des connotations qui portent

${ }^{48}$ Roland, 229, cité dans Centre National de ressources textuelles et lexicales, Étymologie, URL : <http://www.cnrtl.fr/etymologie/sage> (page consultée le 9 mai 2017).

${ }^{49}$ Chrétien de Troyes, Le Conte du Graal, éd. Felix Lecoy, Paris, Champion, 19721975, 2 vol., cité dans Centre National de ressources textuelles et lexicales, Étymologie, URL : <http://www.cnrtl.fr/etymologie/sage> (page consultée le 9 mai 2017).

${ }^{50}$ Voir la note 45.

51 Centre National de ressources textuelles et lexicales, Étymologie, URL : <http://www.cnrtl.fr/etymologie/sage> (page consultée le 9 mai 2017). Sa première attestation remonte aux environs de 1050 avec le sens de «instruit, savant» (Alexis, éd. Christopher Storey, 375). Au XII ${ }^{\mathrm{e}}$ siècle, l'adjectif avait les significations suivantes : «judicieux, intelligent» (Roland, éd. Joseph Bédier, 648); «expérimenté » (ibid., 112); «prudent » (Chanson de Guillaume, éd. Jeanne Wathelet-Willem, 56) ; « juste » (Geffrei Gaimar, Histoire des Anglais, éd. Alexander Bell, 2094).

${ }^{52}$ Le Dictionnaire du Moyen Français, URL: <http://www.atilf.fr/dmf/> (page consultée le 9 mai 2017) : « [...] par le commandement dudit mons. le chancellier, vint et fut present en jugement sur les carreaux, oudit Chastellet, honorable homme et sage maistre Simon Foison, president du roy nostre sire en son parlement " (Registre criminel du Châtelet de Paris du 6 septembre 1389 au 18 mai 1392, éd. Henri DuplèsAgier, Paris, Lahure, 1861, vol. 1).

${ }^{53}$ Ibid. : « [...] Tant soit grant clerc ne sage maistre » (« Miracle de saint Sevestre », dans Miracles de Nostre Dame par personnages, éd. cit., 1878, vol. 3, p. 189-240, cit. p. 219).

${ }^{54}$ La première attestation de maître avec le sens de «personne qui exerce une domination » («celui qui a autorité sur d'autres ») date de 1155 (Wace, Brut, éd. Ivor Arnold, 3120), Centre National de ressources textuelles et lexicales, Étymologie, URL : <http://www.cnrtl.fr/etymologie/maitre> (page consultée le 9 mai 2017).

${ }^{55}$ Le Dictionnaire du Moyen Français, URL: <http://www.atilf.fr/dmf/> (page consultée le 9 mai 2017). 
sur l'état d'esprit de celui qui était élu comme gouverneur, tandis que la copie de Paris reste plus proche de la source latine ${ }^{56}$.

Cependant, dans la majorité des cas, le copiste du manuscrit de Londres fait preuve d'une traduction très littérale et servile de la Vulgate $^{57}$, alors que celui de Paris tente d'améliorer et d'éclaircir son texte avec des paraphrases : «(IV, 5) [...] tut Israel est fesauntz voiz par clamour de joie ${ }^{58}$, et la terre sona ${ }^{59} »$. La copie de Paris spécifie donc le sens initial de «clamore grandi », «grand clamour» dans le manuscrit de Londres ${ }^{60}$. Cette innovation sémantique dans le Livre saint est cohérente avec la trame biblique : le verset 3 du même chapitre illustre tout l'espoir que fonde le peuple d'Israël sur l'arche de l'alliance du Seigneur dont la venue est annoncée au verset en question. Faisant ainsi preuve d'initiative par rapport à la Vulgate ${ }^{61}$, le scribe du manuscrit de Paris démontre qu'il cherche à expliciter les nuances du texte qu'il a sous les yeux.

\footnotetext{
${ }^{56}$ Les exemples présentés sont plutôt exceptionnels pour l'ensemble du corpus, vu que c'est surtout le copiste de Paris qui se permet de temps à autre de dépasser son rôle de traducteur afin de s'approcher de celui d'exégète. Pour plus d'informations sur la traduction comme exégèse, voir Jean Fourquet, «La traduction vue d'une théorie du langage », Langages, ${ }^{\circ}$ 28, La Traduction, dir. Jean-René Ladmiral, 1972, p. 6469.

${ }^{57}$ Voir supra la présence des gloses dans le manuscrit de Londres.

${ }^{58}$ Nous soulignons.

59 «cumque venisset arca foederis Domini in castra vociferatus est omnis Israhel clamore grandi et personuit terra »(I Samuel, IV, 5, Biblia Sacra iuxta Vulgatam Versionem, éd. cit., p. 371), «et lorsque l'arche de l'alliance du Seigneur fut venue dans le camp, tout Israël cria d'un grand cri, et la terre retentit » (La Sainte Bible selon la Vulgate, éd. cit., p. 519).

${ }^{60}$ (IV, 5) «[...] tut Israel cria od grant clamour, et $\left[189 \mathrm{v}^{\circ} \mathrm{a}\right]$ la terre sona ».

${ }^{61}$ Pour la question de l'exégèse chrétienne médiévale, voir les travaux de Gilbert Dahan, Études d'exégèse médiévale. Ancien Testament, Strasbourg, Presses universitaires de Strasbourg, coll. "Écriture et société », 2016, p. 27-72 (chapitre «Genres, formes et méthodes de l'exégèse médiévale »); «Herméneutique et procédures de l'exégèse monastique », dans L'Exégèse monastique au Moyen Âge $\left(X I^{e}-X I V^{e}\right.$ siècle), dir. Gilbert Dahan et Annie Noblesse-Rocher, Paris, Institut d'Études Augustiniennes, coll. «Collection des études augustiniennes. Série Moyen Âge et temps modernes », 2014, p. 115-142.
} 
Si le choix sémantico-lexical permet de mettre en lumière le public visé par des scribes, les différentes graphies des mots peuvent refléter l'impact du contexte de production des copies sur les usages linguistiques. L'origine du copiste ${ }^{62}$, même si elle reste hypothétique, fait partie des facteurs influents. Les exemples de l'édition prouvent que le scribe du manuscrit de Paris se sent manifestement beaucoup plus à l'aise avec la langue française : il recourt plus rarement aux graphies censées être répandues sur le territoire insulaire, qui sont au contraire très utilisées par le copiste du manuscrit de Londres. Ainsi le scribe du manuscrit de Paris préfère-t-il l'utilisation d'une graphie - $e$ pour [e] fermé tonique ${ }^{63}$ à celle généralement proposée par le manuscrit de Londres, $e e^{64}$, et caractérisée comme l'une des possibles graphies anglo-normandes ${ }^{65}$. Les deux manuscrits s'opposent également sur les graphies dans les participes passés où ce son précède la marque du pluriel. Le copiste de Londres préfère alors la variante conservatrice en $-e z$ comme dans «decolpez» $(\mathrm{v}, 4)$, utilisée avant la simplification de l'affriquée [ts $>\mathrm{s}$ ] qui intervient à la fin du XII ${ }^{\mathrm{e}}$ siècle ${ }^{66}$, alors que le scribe du manuscrit de Paris modernise au contraire la transcription en $-e s^{67}$ (que nous transcrivons -és pour les participes).

Le manuscrit de Londres illustre le maintien de la graphie ei, caractéristique de l'anglo-normand, alors que la copie de Paris utilise plus

\footnotetext{
${ }^{62}$ Pierre Nobel, « Gloses anglaises et latines... », art. cit.

${ }^{63}$ Par exemple : cité (I, 3), yvré (I, 14). Le manuscrit présente la graphie e, la transcription par é a été adoptée lors de l'édition.

${ }^{64}$ À comparer avec la note précédente : citee (I, 3), yvree (I, 14).

${ }^{65}$ Mildred Katharine Pope, From latin to modern french with especial consideration of anglo-norman [1934], Manchester, University Press, coll. «Publications of the University of Manchester. French series », 1952, p. 460 (§ 1235).

${ }^{66}$ Ibid., p. 450 (§ 1183).

${ }^{67}$ decopés $(\mathrm{V}, 4)$.
} 
systématiquement $o i$ qui trouve son développement en français médiéval $^{68}$.

Pour transcrire les sons $[\mathrm{u}]$ et [o] nasalisés, le copiste du manuscrit de Londres se sert habituellement du trigraphe $o u n^{69}$ : son emploi est situé chez les scribes anglo-normands qui héritent de l'usage adopté sur le continent à la fin du XII ${ }^{\mathrm{e}}$ siècle $^{70}$.

Concernant la syntaxe, ce sont les constructions participiales qui permettent d'évaluer l'aisance des scribes dans la langue d'arrivée de la traduction $^{71}$ :

${ }^{7}$ sicque faciebat per singulos annos cum redeunte tempore ascenderent templum Domini $[\ldots]^{72}$

ML : (I, 7) Et issint fist el par chescun an, si com il montoient el temple de nostre Sire, le temps returnaunt $[\ldots]$

MP : (I, 7) Et issint fist il par chescun an et, come le temps fust repoiré, qu'il mountast el temple de nostre Seignor $[\ldots]$

${ }^{9}[\ldots]$ et $\quad$ Heli sacerdote sedente super
sellam $[\ldots]^{73}$

ML : (I, 9) [...] Hely, le chapellain, seaunt sur une celle $[\ldots]$

\footnotetext{
${ }^{68}$ ML treis (4 occurrences)/MP trois (2 occurrences), troys (2 occurrences). Pour plus d'informations sur les spécificités des graphies anglo-normandes, voir Ian Short, Manual of Anglo-norman, London, Anglo-norman Text Society, coll. «Anglonorman text society. Occasional publications series », 2007, p. 39.

${ }^{69} \mathrm{ML}$ secounde (2 occurrences)/MP seconde (2 occurrences); ML sount (12 occurrences)/MP sont (10 occurrences); ML toun (1 occurrence) et ton (8 occurrences)/MP ton (8 occurrences).

${ }^{70}$ Mildred Katharine Pope, From latin to modern french with especial consideration of anglo-norman, op. cit., p. 457 (§ 1220).

${ }^{71}$ Nous soulignons.

${ }^{72}$ I Samuel, I, 7, Biblia Sacra iuxta Vulgatam Versionem, éd. cit., p. 366, « c'est ainsi qu'elle faisait chaque année, lorsque, le temps revenant, ils montaient au temple du Seigneur [...] » (La Sainte Bible selon la Vulgate, éd. cit., p. 512).

${ }^{73}$ I Samuel, I, 9, Biblia Sacra iuxta Vulgatam Versionem, éd. cit., p. 366, « [...] et, Héli, le prêtre, étant assis sur son siège [...]» (La Sainte Bible selon la Vulgate, éd. cit., p. 512).
} 
MP : (I, 9) [...] Et Hely, le chapellain, sist sur sa selle [...]

Le scribe du manuscrit de Londres a tendance à proposer une traduction littérale des constructions très courantes en latin à l'ablatif absolu ou avec un simple participe, tandis que le copiste du manuscrit de Paris privilégie leur transformation en prédicats avec le verbe à l'actif, en optant pour une syntaxe plus analytique.

La production et la traduction manuscrites laissent beaucoup de traces révélatrices de facteurs socioculturels. Ainsi la mise en page des copies, de même que la présence des gloses dans le texte, permettent-elles d'établir la distinction entre une Bible destinée à une famille aristocratique (BnF fr. 1) et une Bible qui révèle le travail préparatoire et intellectuel d'un clerc (British Library Royal 1.C.III). Le choix sémantique, tout comme la traduction plus ou moins servile, peuvent également étayer les hypothèses sur les destinataires des copies. Enfin, les traits purement linguistiques, qu'il s'agisse des graphies ou de la syntaxe, font resurgir les facteurs du contexte spatial de l'élaboration des manuscrits.

Le copiste de Londres reste fidèle au texte de la Vulgate qu'il traduit littéralement, mais aussi laborieusement : il utilise des gloses pour transmettre des termes latins avec précision. Apparemment d'origine anglaise, il se conforme souvent à des graphies anglo-normandes. Le scribe du manuscrit de Paris, au contraire, dépasse souvent le simple rôle de traducteur, en recourant à des paraphrases éclairantes. Il propose une version du texte plus mondaine avec des graphies partiellement dédialectalisées, et fait également preuve d'innovation linguistique dans la syntaxe. 
À cette étape, la présente démonstration sert d'ébauche à une analyse linguistique et philologique concernant la composition de la Bible anglo-normande dans son intégralité. L'édition des manuscrits provenant d'un même corpus représente une piste féconde pour les études dans les domaines variés de la médiévistique : c'est la façon dont travaillent les enlumineurs et, surtout, les scribes qui donne la possibilité de faire un petit pas vers les découvertes linguistiques, mais aussi historiques et surtout socioculturelles du monde médiéval. 


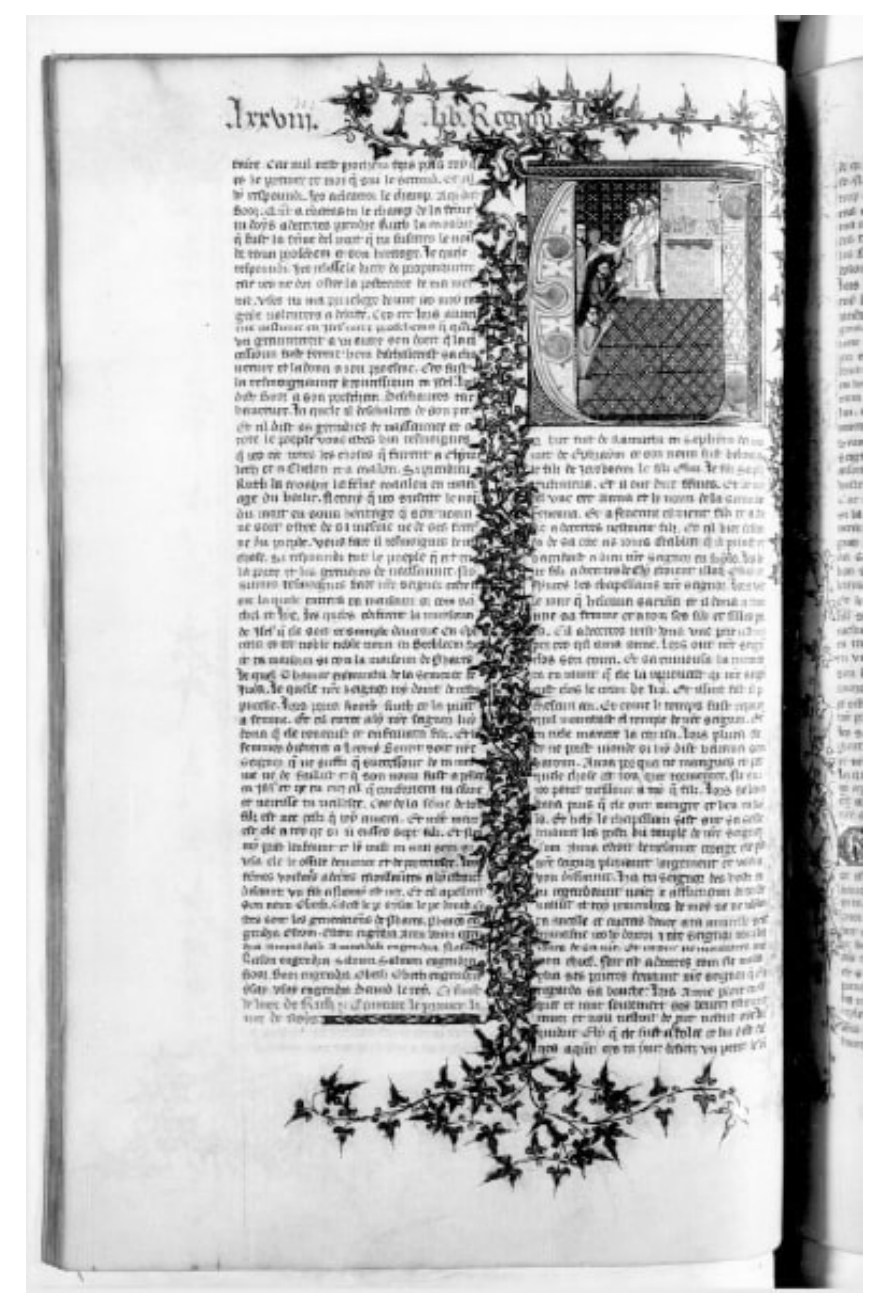

Figure 1 - Première page du Premier Livre de Samuel, Paris, BnF fr. 1 (fol. 78v). 


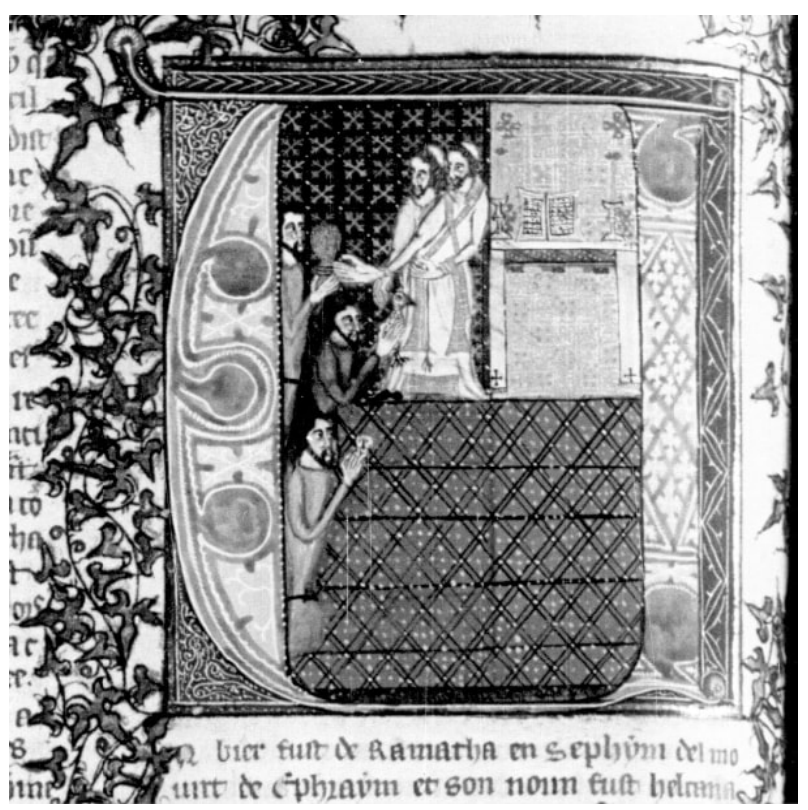

Figure 2 - Grande initiale du Premier Livre de Samuel, Paris, BnF fr. 1 (fol. $7_{8 v^{\circ}} b$ ).

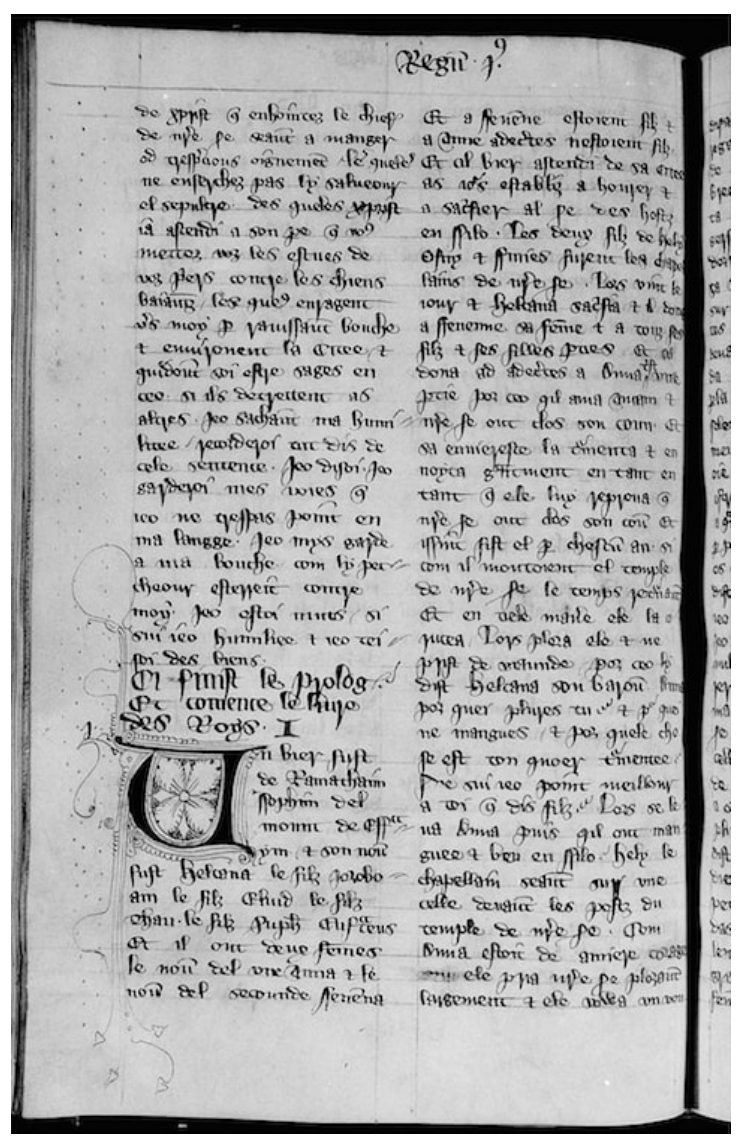

Figure 3 - Première page du Premier Livre de Samuel, Londres, British Library Royal 1.C.III (fol. 186v). 


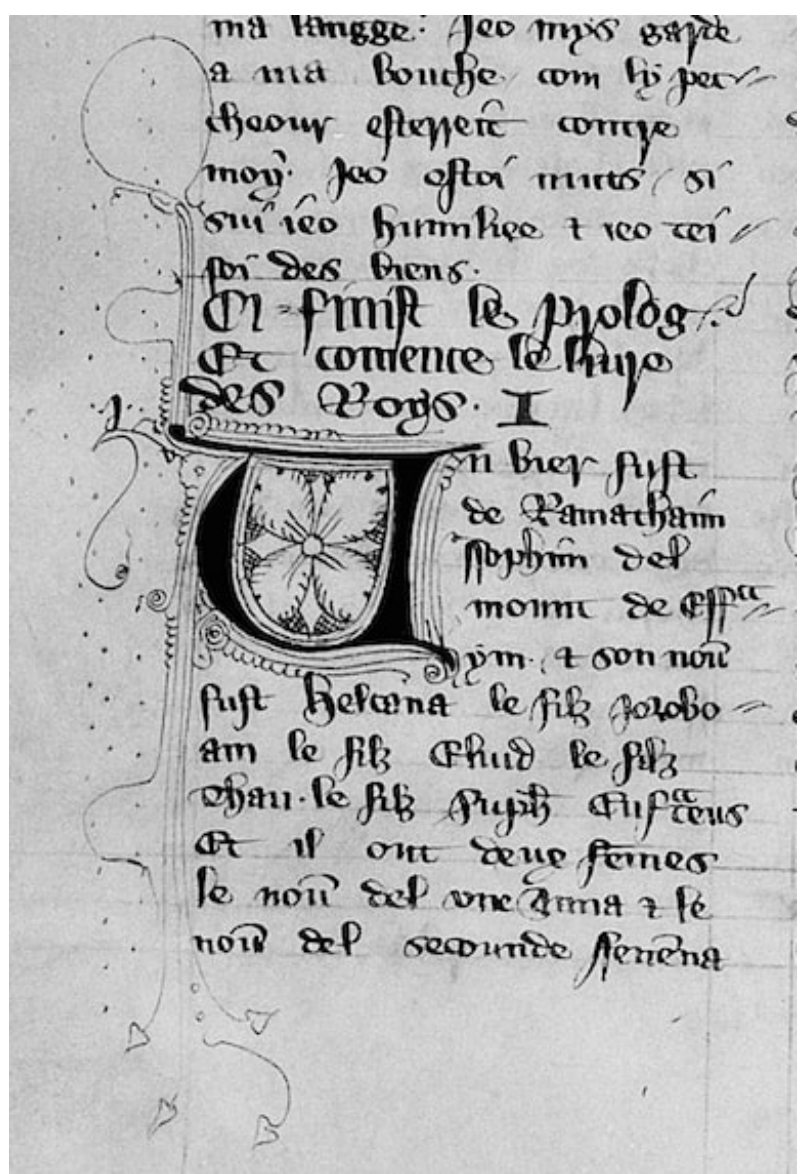

Figure 4 - Grande initiale du Premier Livre de Samuel, Londres, British Library Royal 1.C.III (fol. $186 v^{\circ}$ a). 